\title{
REMARKS ON SOME FIXED POINT THEOREMS
}

\author{
TECK-CHEONG LIM
}

\begin{abstract}
A compact Hausdorff pseudo-topology is introduced on every closed convex bounded subset of a uniformly convex Banach space and is used to prove a previous theorem of the author.
\end{abstract}

In [7], we used a transfinite induction method which depends on the structure of the real line to prove the following fixed point theorem for multivalued nonexpansive mappings:

THEOREM 1. Let $K$ be a closed convex bounded nonempty subset of a uniformly convex Banach space and let $T: K \rightarrow \mathfrak{C}(K)$ be a nonexpansive mapping, where $e(K)$ denotes the family of nonempty compact (not necessarily convex) subsets of $K$, equipped with the Hausdorff metric. Then $T$ has a fixed point, i.e. there exists $x \in K$ such that $x \in T x$.

The properties of real numbers we used are the order property and the separability, or more explicitly, that a decreasing nonnegative transfinite sequence indexed by ordinals less than the uncountable ordinal $\Omega$ must be eventually constant.

Recently, Caristi and Kirk [1], [2], [5] have proven the following theorem and have given several interesting applications:

Theorem 2 [1], [2]. Let $X$ be a complete metric space, and let $g: X \rightarrow X$ be a self-map of $X$. Suppose that there exists a lower semicontinuous nonnegative real-valued mapping $\xi$ such that for all $x$ in $X$,

$$
d(x, g(x)) \leqslant \xi(x)-\xi(g(x)) .
$$

Then $g$ has a fixed point.

Chi Song Wong [8] has given a simple proof of the Caristi-Kirk theorem by using the transfinite induction method mentioned above. On the other hand, we define a pseudo-compact-Hausdorff-topology on any closed convex bounded subset of a uniformly convex Banach space and give Theorem 1 a simpler and more conceptual proof. It is our feeling that the existence of such a compact Hausdorff pseudo-topology may well serve to explain the similarity between uniform convexity and compactness in some aspects of geometric fixed point theory.

Presented to the Society, January 20, 1975 under the the title $A$ remark on a fixed point theorem; received by the editors September 5, 1975.

AMS (MOS) subject classifications (1970). Primary 46A05.

Copyright (9) 1977, American Mathematical Society 
Let $(X, d)$ be a metric space. A sequence $\left\{x_{n}\right\}$ in $X$ is said to $\Delta$-converge to a point $x \in X$, written $x_{n} \rightarrow^{\Delta} x$, if

$$
\limsup _{i} d\left(x_{n_{i}}, x\right) \leqslant \limsup _{i} d\left(x_{n_{i}}, y\right)
$$

for every subsequence $\left\{x_{n_{i}}\right\}$ of $\left\{x_{n}\right\}$ and every $y \in X$. In the terminology of asymptotic center (Edelstein [3], Lim [6]), this says that $x$ is an asymptotic center of every subsequence of $\left\{x_{n}\right\} .\left\{x_{n}\right\}$ is said to $\Delta$-converge strongly to $x$ if

$$
\lim d\left(x_{n}, x\right) \leqslant \lim \inf d\left(x_{n}, y\right) \text { for every } y \in X .
$$

This is equivalent to saying that all subsequences of $\left\{x_{n}\right\}$ have a common asymptotic center $(=x)$ and asymptotic radius $\left(=\lim d\left(x_{n}, x\right)\right)$. In general, $x$ is not unique. That strong $\Delta$-convergence implies $\Delta$-convergence is obvious.

$\Delta$-convergence has the following properties of which only the first two are satisfied by strong $\Delta$-convergence:

(i) if $x_{n}=x$ for every $n$, then $x_{n} \rightarrow^{\Delta} x$;

(ii) if $x_{n} \rightarrow^{\Delta} x$, then every subsequence of $\left\{x_{n}\right\} \Delta$-converges to $x$;

(iii) if $\left\{x_{n}\right\}$ does not $\Delta$-converge to $x$, then there exists a subsequence of which every subsequence does not $\Delta$-converge to $x$.

Clearly these definitions and properties can be formulated for nets. A set $X$ equipped with a convergence class satisfying (i), (ii) and (iii) (or only (i) and (ii)) will be called a pseudo-topological space and the convergence class will be called a pseudo-topology on $X$. Note that we need only one additional axiom to define topological spaces by convergence classes, see e.g., Kelley [4]. By using nets, concepts in topological spaces can be carried over to quasitopological spaces. Thus a quasi-topological space is compact if every net in it has a convergent subnet and is Hausdorff if a net can converge to at most one point. In what follows, (strong) $\Delta$-topology will refer to the quasi-topology with the convergence class given by sequences satisfying (2) ((3) respectively). One may replace "sequences" by "nets" in this definition and will obtain the same conclusions of Theorems 3 and 4 below.

A metric space is said to be $\Delta$-complete if for every bounded sequence (or net) $\left\{x_{n}\right\}$ in $X$ there is an $x \in X$ such that

$$
\lim _{n} \sup d\left(x_{n}, x\right) \leqslant \lim _{n} \sup d\left(x_{n}, y\right)
$$

for every $y \in X$ i.e. $\left\{x_{n}\right\}$ has an asymptotic center in $X$.

THEOREM 3. Every bounded $\Delta$-complete metric space $X$ is strongly $\Delta$-compact (and hence $\Delta$-compact), i.e. every sequence in $X$ has a strongly $\Delta$-convergent subsequence.

THEOREM 4. Every closed convex bounded subset of a uniformly convex Banach space is compact Hausdorff under the (strong) $\Delta$-topology.

Our proofs of Theorems 3 and 4 need the following set-theoretical result. For two sequences $\left\{x_{n}\right\}$ and $\left\{y_{n}\right\}$ in a set, let us say $\left\{x_{n}\right\}$ is an essential 
subsequence of $\left\{y_{n}\right\}$ if there exists a positive integer $N$ such that $\left\{x_{n}\right\}_{n \geqslant N}$ is a subsequence of $\left\{y_{n}\right\}$.

Proposition 1. Let $X$ be a set and let $\left\{x_{n}\right\}$ be a sequence in $x$. Let $r$ be $a$ real-valued function whose domain is the set of subsequences of $\left\{x_{n}\right\}$. Suppose $r(y) \leqslant r(z)$ whenever $y$ is an essential subsequence of $z$. Then there is $a$ subsequence $w$ of $\left\{x_{n}\right\}$ such that $r(z)=r(w)$ for every subsequence $z$ of $w$.

Proof. Denote by $\widetilde{F}$ the family of subsequences of $\left\{x_{n}\right\}$. Define an ordering $\leqslant$ on $\%$ as follows:

For $x, y \in \mathscr{F}$, we put $x<y$ if $x$ is an essential subsequence of $y$ and $r(x)<r(y)$. Then we say $x \leqslant y$ if $x<y$ or $x$ is identically equal to $y$.

It is easy to check that $\leqslant$ is a reflexive, antisymmetric and transitive relation. Let $\mathcal{E}$ be a chain in $\mathscr{F}$. Let $r=\inf \left\{r(x): x \in \mathcal{E}^{\prime}\right\}$. If there is an $x \in \mathcal{E}$ such that $r(x)=r$, then $x$ is a lower bound for $\mathcal{E}$. Therefore we assume that such $x$ does not exist. Let $x_{n}$ be a sequence in $E$ such that $r\left(x_{n}\right)$ strictly decreases to $r$. Since $e$ is a chain, we must have $x_{1}>x_{2}>\ldots$ By using the diagonal process, dropping a finite number of terms in each sequence $X_{n}$ if necessary, we obtain a sequence $y$ which is an essential subsequence of $x_{n}$ for every $n$. Then, by assumption, $r(y)<r\left(x_{n}\right)$ for every $n$ and, hence, $r(y)<r(x)$ for every $x \in \mathcal{E}$. Since each $x \in \mathcal{E}$ is an essential subsequence of $x_{n}$ for some $n$, we conclude that $y$ is a lower bound for $E$.

By Zorn's lemma, $\widetilde{y}$ has a minimal element $z$. Let $w$ be a subsequence of $z$. Then $r(w) \leqslant r(z)$. If $r(w)<r(z)$, then $w \leqslant z$ and by the minimality and antisymmetry we must have $w-z$ which implies $r(w)=r(z)$, a contradiction. Hence $r(w)=r(z)$. Q.E.D.

Proof of Theorem 3. Let $\left\{x_{n}\right\}$ be a sequence in $X$. For every subsequence $\left\{x_{n_{i}}\right\}$, let

$$
r\left(\left\{x_{n_{i}}\right\}\right)=\inf \left\{\limsup _{i} d\left(x_{n_{i}}, y\right): y \in X\right\} .
$$

By Proposition $1\left\{x_{n}\right\}$ contains a subsequence which we still denote by $\left\{x_{n}\right\}$ such that

$$
r\left(\left\{x_{n}\right\}\right)=r\left(\left\{x_{n_{i}}\right\}\right)=r
$$

for every subsequence $\left\{x_{n_{i}}\right\}$ of $\left\{x_{n}\right\}$. By $\Delta$-completeness, there exists an $x \in X$ such that

$$
\lim \sup d\left(x_{n}, x\right)=r\left(\left\{x_{n}\right\}\right) .
$$

For every subsequence $\left\{x_{n_{i}}\right\}$ of $\left\{x_{n}\right\}$, we have

$$
\begin{aligned}
\lim \sup d\left(x_{n_{i}}, x\right) & \leqslant \lim \sup d\left(x_{n}, x\right) \\
& =r\left(\left\{x_{n}\right\}\right)=r\left(\left\{x_{n_{i}}\right\}\right) \leqslant \lim \sup d\left(x_{n_{i}}, x\right) ;
\end{aligned}
$$

thus

$$
\lim \sup d\left(x_{n_{i}}, x\right)=r\left(\left\{x_{n_{i}}\right\}\right)
$$


This shows that all subsequences $\left\{x_{n_{i}}\right\}$ of $\left\{x_{n}\right\}$ have a same asymptotic center $x$ and a same asymptotic radius $r$. Q.E.D.

Proof of Theorem 4. This follows from Theorem 3 and the uniqueness of asymptotic center as proved by Edelstein [3]. Q.E.D.

Let us now give

Proof of Theorem 1. By a standard argument, there exist sequences $\left\{x_{n}\right\}$ and $\left\{y_{n}\right\}$ such that $y_{n} \in T x_{n}$ and $\left\|x_{n}-y_{n}\right\| \rightarrow 0$. By Theorem $4,\left\{x_{n}\right\}$ has a $\Delta$-convergent subsequence which we still denote by $\left\{x_{n}\right\}$. Let $x$ be its $\Delta$-limit. We assert that $x \in T x$. For each $n$, choose $p_{n} \in T x$ such $\left\|p_{n}-y_{n}\right\| \leqslant \| x-$ $x_{n} \|$. Since $T x$ is compact, there exists a convergent subsequence $\left\{p_{n_{i}}\right\}$ of $\left\{p_{n}\right\}$ such that $p_{n_{i}} \rightarrow p$ for some $p \in T x$. It can be easily shown, by using $\left\|x_{n}-y_{n}\right\|$ $\rightarrow 0,\left\|p_{n}-y_{n}\right\| \leqslant\left\|x-x_{n}\right\|$ and $x_{n} \rightarrow^{\Delta} x$, that $x_{n_{i}} \rightarrow^{\Delta} p$. Since also $x_{n_{i}} \rightarrow^{\Delta} x$, we must have $x=p \in T x$ by the uniqueness of $\Delta$-limit. Q.E.D.

REMARK. I am indebted to the referee for informing me that K. Goebel has discovered independently a similar proof of Theorem 1 in a paper published in Ann. Univ. Mariae Curie-Skłodowska.

\section{REFERENCES}

1. J. Caristi, Fixed point theorems for mappings satisfying inwardness conditions (to appear).

2. J. Caristi and W. A. Kirk, Geometric fixed point theory and inwardness conditions (to appear).

3. M. Edelstein, Fixed point theorems in uniformly convex Banach spaces, Proc. Amer. Math. Soc. 44 (1974), 369-374. MR 50 \# 10917.

4. J. L. Kelley, General topology, Van Nostrand, Princeton, N. J., 1955. MR 16, 1136.

5. W. A. Kirk and J. Caristi, Mapping theorems in metric and Banach spaces (to appear).

6. T. C. Lim, Characterizations of normal structure, Proc. Amer. Math. Soc. 43 (1974), 313-319. MR 50 \# 14173.

7. Banach space, Bull. Amer. Math. Soc. 80 (1974), 1123-1126.

8. Chi Song Wong, On a fixed point theorem of contractive type, Proc. Amer. Math. Soc. 57 (1976), 283-284.

Department of Mathematics, University of Chicago, Chicago, Illinois 60637

Current address: 61-C Lorong Kumara, Singapore 27, Singapore 